\title{
Infrared behaviors of SU(2) gauge theory
}

\author{
Kimmo Tuominen ${ }^{1,2, a}$ \\ ${ }^{1}$ Department of Physics \& \\ ${ }^{2}$ The Helsinki Institute of Physics, \\ P.O.Box 64, FI-00014, University of Helsinki, Finland
}

\begin{abstract}
We will discuss some recent results in the determination of the location of the conformal window in SU(2) gauge theory with $N_{f}$ fermions in the fundamental representation of the gauge group. In particular, we will demonstrate that the long distance behavior of the continuum theory with $N_{f}=6$ is governed by an infrared stable fixed point.
\end{abstract}

\section{Introduction}

Determination of the vacuum phase of an $\mathrm{SU}(N)$ gauge theory with $N_{f}$ massless flavors of Dirac fermions provides a basic problem for our current understanding of perturbative and nonperturbative gauge theory dynamics. For example, in the case of fermions transforming in the fundamental representation of the gauge group the following quantitative features have been established: At small $N_{f}$ the theory breaks chiral symmetry of the vacuum similarly as QCD, while above $N_{f}=11 \mathrm{~N} / 2$ the theory is not asymptotically free. Between $N_{f}^{(c)} \leq N_{f} \leq 11 N / 2$, inside the so-called conformal window, the long distance behaviour is expected to become governed by a nontrivial infrared stable fixed point (IRFP), and the vacuum phase of the theory has infrared conformal behavior.

The IRFP in typical cases is at strong coupling, making the determination of the lower boundary of the conformal window $N_{f}^{(c)}$ challenging. Nonperturbative methods are required, and over recent years a lots of efforts in the field of lattice gauge theory has been devoted to address these questions. One particular benchmark theory which has been thoroughly investigated is $\mathrm{SU}(2)$ gauge theory with two Dirac fermions in the adjoint representation [1-3]. This theory shows clear indications for the existence of an IRFP.

Here we focus on SU(2) gauge theory with $N_{f}$ fermions in the fundamental representation of the gauge group. Systematic investigation of some of these theories has been carried out in [4-8]. For few flavors the theory is expected to be QCD-like and this is confirmed by the simulations as well as the existence of a fixed point for ten flavors compatibly with perturbation theory. However, at intermediate number of flavors, in particular at $N_{f}=6$ the results remain inconclusive so far.

\section{Lattice formulation}

We study the SU(2) gauge theory with Dirac fermions in the fundamental representation of the gauge group. In the lattice formulation we use the HEX-smeared [9] clover improved Wilson fermion action

\footnotetext{
a e-mail: kimmo.i.tuominen@helsinki.fi
} 
with partially smeared plaquette gauge action $S=\left(1-c_{g}\right) S_{G}(U)+c_{g} S_{G}(V)+S_{F}(V)$, Here $U$ and $V$ are, respectively, the unsmeared and smeared gauge link matrices. $S_{G}$ is the standard single plaquette Wilson gauge action for the SU(2) Yang Mills theory, where $\beta_{L}=4 / g_{0}^{2}, c_{g}$ is the mixing parameter between the smeared and unsmeared plaquettes, and $a$ is the lattice spacing. Using the partially smeared gauge action helps to avoid unphysical bulk phase transitions at strong coupling [10]. We set the gauge action mixing parameter to the value $c_{g}=0.5$; for the details of this smearing, see [3].

The clover improved Wilson fermion action is

$$
S_{F}=a^{4} \sum_{\alpha=1}^{N_{f}} \sum_{x}\left[\bar{\psi}_{\alpha}(x)\left(i D+m_{0}\right) \psi_{\alpha}(x)+a c_{\mathrm{sw}} \bar{\psi}_{\alpha}(x) \frac{i}{4} \sigma_{\mu \nu} F_{\mu v}(x) \psi_{\alpha}(x)\right],
$$

where $D$ is the standard lattice Wilson-Dirac operator,

$$
D=\frac{1}{2}\left[\gamma_{\mu}\left(\nabla_{\mu}^{*}+\nabla_{\mu}\right)-a \nabla_{\mu}^{*} \nabla_{\mu}\right]
$$

where $\nabla_{\mu}\left(\nabla_{\mu}^{*}\right)$ is the gauge covariant forward (backward) lattice derivative

$$
\nabla_{\mu} \psi(x)=\frac{1}{a}\left[V_{\mu}(x) \psi(x+a \hat{\mu})-\psi(x)\right]
$$

defined in terms of the smeared link matrices. The last term in the fermion action is the clover term containing the usual symmetrized field strength tensor. To removes $O(a)$ errors from on-shell quantities we set the Sheikholeslami-Wohlert coefficient $c_{\mathrm{sw}}$ to its tree-level value $c_{\mathrm{sw}}=1$. At the temporal boundaries we use Dirichlet boundary conditions with the gauge link matrices set to unity and fermion fields to zero, while the spatial boundaries are taken periodic. These bounday conditions remove the fermion zero modes and allow us to run simulations at vanishing physical quark masses.

The Wilson fermion action breaks the chiral symmetry explicitly and requires additive renormalization of the quark mass. We tune to the chiral limit by requiring the vanising of the PCAC quark mass $a M(L / 2)$, defined by the relation [11]

$$
M\left(x_{0}\right)=\frac{1}{4} \frac{\left(\partial_{0}^{*}+\partial_{0}\right) f_{A}\left(x_{0}\right)}{f_{P}\left(x_{0}\right)},
$$

where $f_{A}$ and $f_{P}$ are axial and pseudoscalar current density correlations. In principle there is an $O(a)$ improvement term to this equation [11], but our use of the smeared gauge links renders its contribution very small and we ignore it here.

\section{Results}

The vacuum spectrum of the theory provides a simple probe of the nonperturbative properties of the theory, especially in the case of QCD-like dynamics where the results can be straightforwardly interpreted. The masses of color singlet meson states are determined by fitting the time sliced average correlation functions with Coulomb gauge fixed wall sources.

For two flavors, in the limit of vanishing quark mass, the spectrum behaves as expected on the basis of spontaneously broken chiral symmetry: the mass of the pseudoscalar state scales towards zero while the mass of the vector state has a finite intercept. These behaviors are shown in the left panel of Fig. 1, while the right panel shows the pseudoscalar decay constant $F_{\pi}$ as a function of the quark mass. 

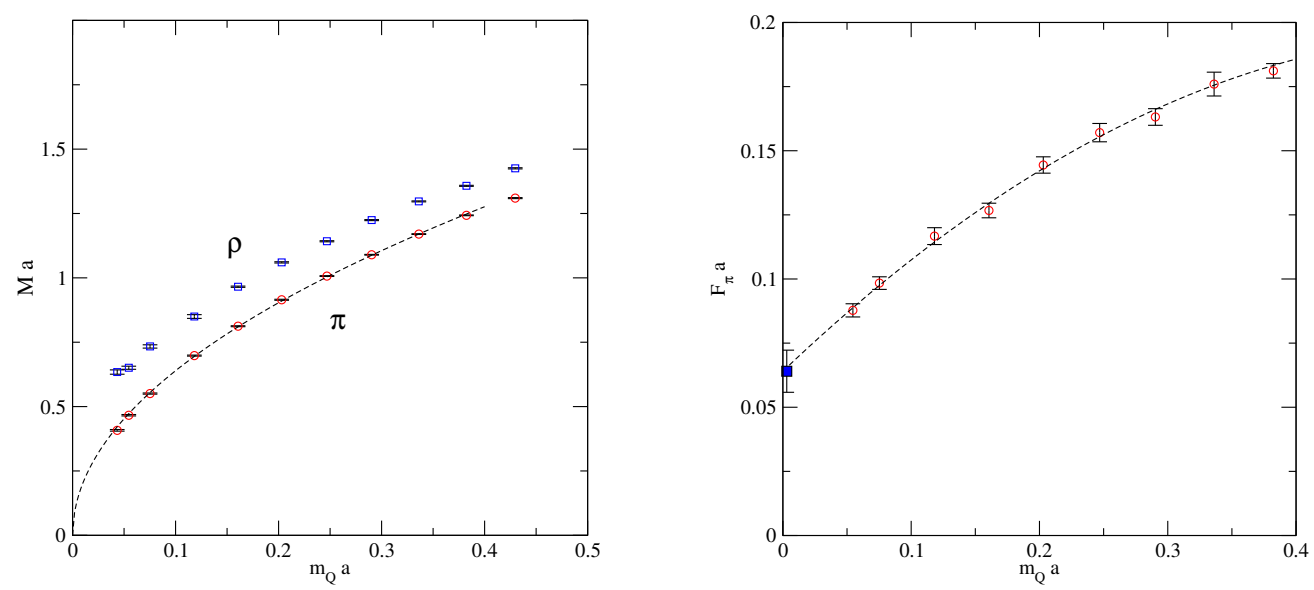

Figure 1. The vacuum spectra of SU(2) gauge theory with two Dirac flavors in the fundamental representation. Left: the masses of the pseudoscalar and vector states as a function of the quark mass. Right: the pseudoscalar decay constant as a function of the quark mass.

The interpretation of the results from the spectral measurements become more uncertain when approaching the conformal window. In Fig. 2 we show the results for the measurement of the spectrum in the four-flavor theory. While the results are consistent with the qualitatively similar behavior as in the two-flavor case, the pseudoscalar decay constant is compatible with a finite or zero value as $m_{Q}$ approaches zero.
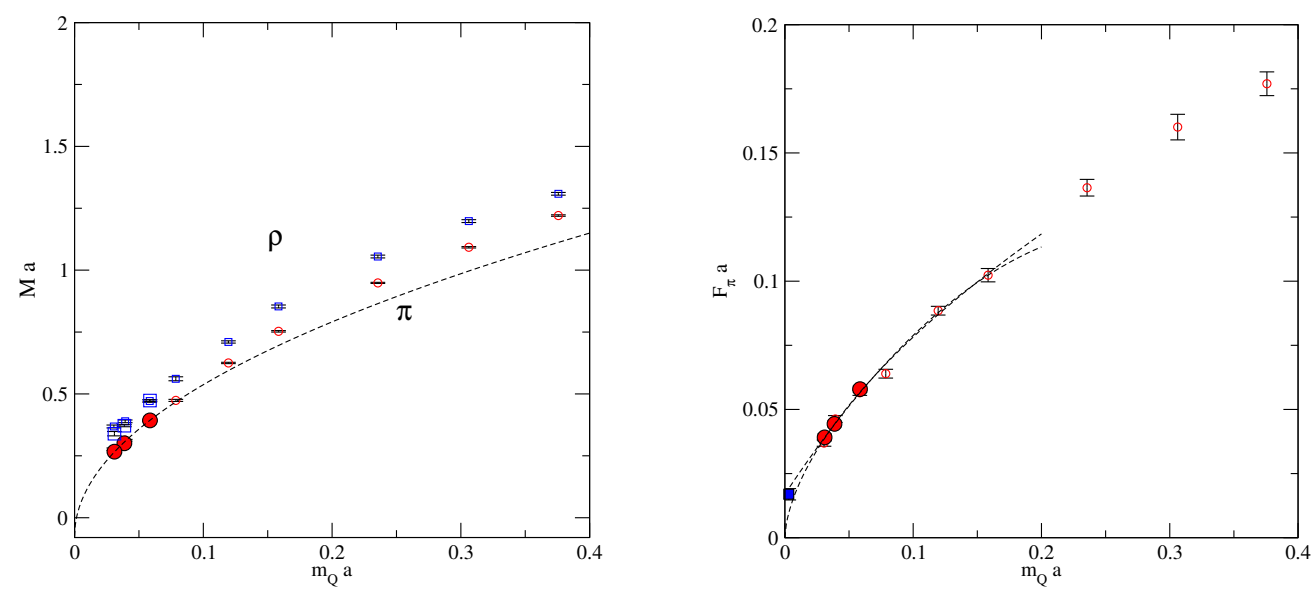

Figure 2. Vacuum properties of SU(2) gauge theory with four Dirac flavors in the fundamental representation. Left: the masses of the pseudoscalar and vector states as a function of the quark mass. Right: the pseudoscalar decay constant as a function of the quark mass.

The difficulties become increasingly profound as $N_{f}$ is increased further: In Fig. 3 we show the masses of the pseudoscalar and vector states measured at two different volumes. The results show 
strong finite volume effects when approaching the chiral limit. Extrapolating the results seems to imply that the masses are scaling equivalently towards zero, which could be taken as an indication of a conformal behavior, i.e. a signal of the existence of a nontrivial fixed point.

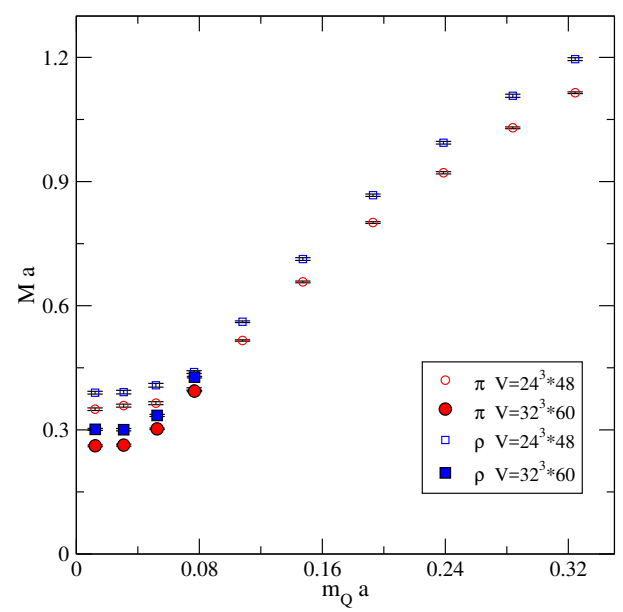

Figure 3. The masses of the pseudoscalar and vector states in an SU(2) gauge theory with four Dirac flavors in the fundamental representation. The results correspond to lattice coupling at two different volumes.

To make more definitive statement on the existence of a fixed point, we measure the running of the coupling using the Yang-Mills gradient flow [12] combined with the Schrödinger functional finitevolume scaling [13]. To set up this computation, we introduce an extra coordinate, the flow time $t$, and a flow gauge field $B_{\mu}(x ; t)$, which evolves according to

$$
\partial_{t} B_{\mu}=D_{\nu} G_{v \mu}
$$

where $G_{\mu \nu}(x ; t)$ is the field strength of the flow field $B_{\mu}$ and $D_{\mu}=\partial_{\mu}+\left[B_{\mu}, \cdot\right]$. The initial condition is defined in terms of the original continuum gauge field $A_{\mu}$ such that $B_{\mu}(x ; t=0)=A_{\mu}(x)$.

The flow smooths the gauge field over the radius $r \sim \sqrt{8 t}$ and renormalizes gauge invariant observables [14]. Therefore the field strength of $\mathrm{SU}(N)$ gauge theory, which to leading order in perturbation theory evolves as [15]

$$
\langle E(t)\rangle=-\frac{1}{4}\left\langle G_{\mu \nu} G_{\mu \nu}\right\rangle(t)=\frac{3\left(N^{2}-1\right) g_{0}^{2}}{128 \pi^{2} t^{2}}+O\left(g_{0}^{4}\right),
$$

can be used to define the coupling at scale $\mu=1 / \sqrt{8 t}$ nonperturbatively as

$$
g_{\mathrm{GF}}^{2}(\mu)=\left.\frac{128 \pi^{2}}{3\left(N^{2}-1\right)} t^{2}\langle E(t)\rangle\right|_{t=1 / 8 \mu^{2}} .
$$

In the lattice formulation of SU(2) theory, the continuum flow field is replaced by the lattice link variables $U_{\mu}(x ; t)$. These are then evolved according to

$$
\frac{\partial}{\partial t} U_{\mu}(x ; t)=-g_{0}^{2}\left(\frac{\partial}{\partial U_{\mu}(x ; t)} S_{\mathrm{GF}}[U]\right) U_{\mu}(x ; t)
$$


with the initial condition $U_{\mu}(x ; t=0)=U_{\mu}(x)$. For the action $S_{\mathrm{GF}}$ appearing in the flow evolution equation, we use the Lüscher-Weisz pure gauge theory action [16].

In order to minimize both lattice artifacts and finite volume effects in (7), the scale must be limited into a regime $1 / L \ll \mu \ll 1 / a$. As detailed in [13], we relate the lattice scale to the renormalization scale by defining a dimensionless parameter $c_{t}$ such that

$$
\mu^{-1}=c_{t} L=\sqrt{8 t}
$$

Each value of $c_{t}$ therefore defines a particular scheme for the coupling. A range of $c_{t}=0.3-0.5$ is suggested in [13] as within this range the cutoff effects, statistical variance, and boundary effects [17], are reasonably small. We use $c_{t}=0.4$ in our analysis, but we have studied the dependence of our results on the variation of $c_{t}$.

The running of the coupling is quantified using the lattice step scaling function $\Sigma(u, s, L / a)$ and its continuum limit $\sigma(u, s)$. These describe the change of the measured coupling when the linear size of the system is increased from $L$ to $s L$, while keeping the bare coupling $g_{0}^{2}$, and consequently the lattice spacing, constant:

$$
\begin{aligned}
& \Sigma(u, s, L / a)=\left.g_{\mathrm{GF}}^{2}\left(g_{0}^{2}, s L / a\right)\right|_{g_{\mathrm{GF}}^{2}\left(g_{0}^{2}, L / a\right)=u} \\
& \sigma(u, s)=\lim _{a / L \rightarrow 0} \Sigma(u, s, L / a) .
\end{aligned}
$$

For the eight flavor theory we choose $s=2$.

A proper continuum extrapolation would require the step scaling function to be evaluated at fixed value of the coupling. However, in practice the simulations are done at a selected fixed set of bare couplings $\beta_{L}=4 / g_{0}^{2}$, which do not correspond to same $g_{\mathrm{GF}}^{2}$-values when step scaling in (10) is measured at different $L / a$. It is therefore necessary to interpolate the $g_{0}^{2}$-dependence of the actual measurements of $g_{\mathrm{GF}}^{2}\left(g_{0}^{2}, L / a\right)$ at each lattice size $L / a$. We use a rational interpolating function [6]:

$$
g_{\mathrm{GF}}^{2}\left(g_{0}^{2}, L / a, t\right)=g_{0}^{2} \frac{1+\sum_{i=1}^{n} a_{k} g_{0}^{2 i}}{1+\sum_{i=1}^{m} b_{k} g_{0}^{2 i}},
$$

with $n=7$ and $m=1$, which were determined to give a reasonable $\chi^{2} /$ d.o.f.

We expect the dominant discretization errors to be of order $a^{2}$. The continuum extrapolation of the step scaling function $\Sigma(u, 2, L / a)$, defined in Eq. (10), is then performed by using a quadratic extrapolation function

$$
\Sigma(u, 2, L / a)=\sigma(u, 2)+c(u)(L / a)^{-2}
$$

on lattices of size $L / a=10,12,16$. Furthermore, to minimize the cutoff effects in the gradient flow coupling $g_{\mathrm{GF}}^{2}$ [18] a tunable $O\left(a^{2}\right)$ correction $\tau_{0}$ is implemented, as suggested in ref. [19]:

$$
g_{\mathrm{GF}}^{2}=\frac{t^{2}}{\mathcal{N}}\left\langle E\left(t+\tau_{0} a^{2}\right)\right\rangle=\frac{t^{2}}{\mathcal{N}}\langle E(t)\rangle+\frac{t^{2}}{\mathcal{N}}\left\langle\frac{\partial E(t)}{\partial t}\right\rangle \tau_{0} a^{2}+O\left(a^{4}\right) .
$$

This is applied at the beginning of the analysis, i.e. for each new choice of $\tau_{0}$ the coupling $g_{\mathrm{GF}}$ and step scaling are recalculated.

As long as $\left|\tau_{0}\right| \ll t / a^{2}$, the $\tau_{0}$-correction will have a relatively small effect in the continuum [20]. The cutoff effects grow as a function of a coupling making the $\tau_{0}$-correction dependent on the coupling, $\tau_{0}\left(g_{\mathrm{GF}}^{2}\right)$. Note that for a consistent continuum limit the functional dependence must be on $g_{\mathrm{GF}}^{2}$ 

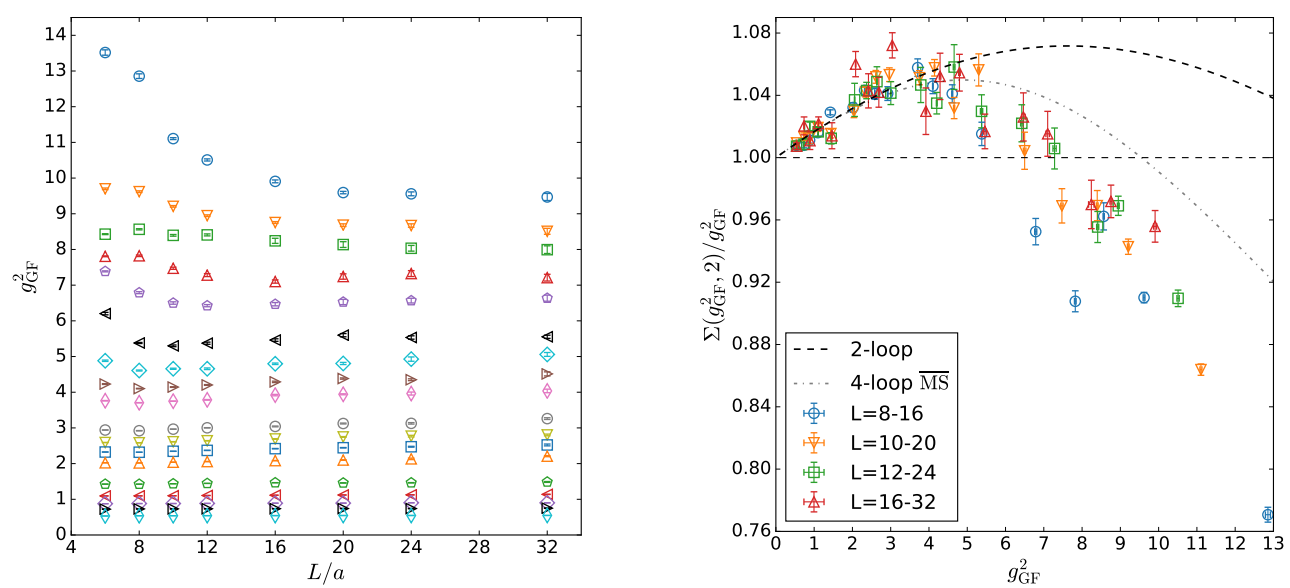

Figure 4. Left: The measured values of $g_{\mathrm{GF}}^{2}\left(g_{0}, L / a\right)$. Right: The lattice step scaling function.
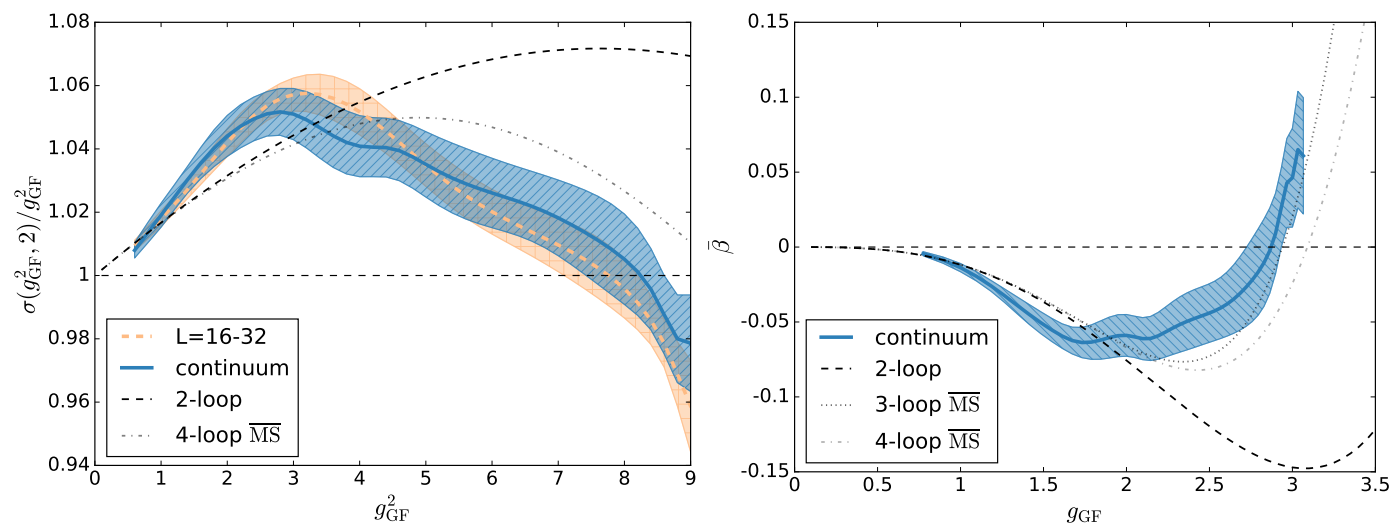

Figure 5. Left: The scaled step scaling function $\sigma\left(g_{\mathrm{GF}}^{2}, 2\right) / g_{\mathrm{GF}}^{2}$, with continuum extrapolation done using the $10-20,12-24$ and $16-32$ volume pairs. Right: The estimate of $\beta$-function.

instead of $g_{0}^{2}$ [21]. In our case, at $c_{t}=0.4$, we have found that a good result can be obtained with the functional form

$$
\tau_{0}=0.06 \log \left(1+g_{\mathrm{GF}}^{2}\right),
$$

where the logarithm regulates the behaviour of $\tau_{0}$ at strong coupling. In order to reach the final $\tau_{0}$ and $g_{\mathrm{GF}}^{2}$ we calculate the correction iteratively starting from the bare coupling $g_{0}^{2}$.

The measured values of running coupling at $c_{t}=0.4$ with the $\tau_{0}$ correction taken into account are shown in the left panel of Fig. 4. Substantial finite volume effects are observed on smaller lattices at around $g^{2} \equiv g_{\mathrm{GF}}^{2} \approx 8$. On the right panel of the figure the scaled step scaling function $\Sigma\left(g^{2}, 2, L / a\right) / g^{2}=g^{2}\left(g_{0}^{2}, 2 L / a\right) / g^{2}\left(g_{0}^{2}, L / a\right)$ is shown at $L / a=8,10,12$ and 16 . In the weak coupling region the running follows closely the universal two loop perturbative curve up to $g^{2}=4$, but then deviates towards a possible fixed point around $g^{2}=8$. Also shown in the figure is the four loop $\overline{\mathrm{MS}}$ 
result, but it should be noted that this result is scheme dependent and cannot be directly compared with our result.

In the left panel of Fig. 5 we show the continuum limit of the step scaling function (13) using the $\tau_{0}$ correction from (15) and step scaling volume pairs 10-20, 12-24 and 16-32. The pair $8-16$, which shows strong finite volume effects, is excluded. Also the continuum extrapolation with the largest volume step scaling function is shown in the figure. This can be compared to the uninterpolated step scaling presented in Fig. 4. The error bands shown in the figure include only the statistical errors from the measurements, interpolation and extrapolation and the error propagation has been done by jackknife blocking throughout the whole analysis. The variation between these two curves provides an estimate of the systematic errors in the extrapolation, and seem to be well in control.

We have also studied the dependence of the continuum extrapolation on the $\tau_{0}$-correction, value of the $c_{t}$-parameter in the lattice action and on the different lattice discretizations of the energy observable $E(t)$. We find that the continuum extrapolated results are remarkably robust against all these variations. As a final result, in the $c_{t}=0.4$ scheme, the fixed point coupling is located at $7 \lesssim g_{*}^{2} \lesssim 9$, where the error is dominated by the systematics of the extrapolation.

The step scaling function is related to the $\beta$-function as

$$
-2 \ln (s)=\int_{u}^{\sigma(u, s)} \frac{d x}{\sqrt{x} \beta(\sqrt{x})} .
$$

Close to the fixed point, where the running is slow and $|\beta|$ small, we can approximate the $\beta$-function by

$$
\beta(g) \approx \bar{\beta}(g)=\frac{g}{2 \ln (s)}\left(1-\frac{\sigma\left(g^{2}, s\right)}{g^{2}}\right) .
$$

From the step scaling function $\sigma\left(g_{\mathrm{GF}}^{2}\right)$ we can construct the approximate beta function $\bar{\beta}(g)$ using Eq. (17), and this is shown in the right panel of Fig. 5.

These behaviors confirm that the theory with eight fundamental flavors should be well within the conformal window. We have also performed a similar analysis of the evolution of the coupling for the six-flavor theory, which on the basis of the measurement of the spectrum and earlier studies on the evolution of the coupling remains inconclusive.

The analysis in the six flavor theory follows closely the eight flavor case and we only quote the essential features here before presenting the results. The gradient flow is evolved with Lüscher-Weisz action, clover definition of the energy density and with the choice $c_{t}=0.3$. We run the simulations using lattice sizes $8,12,16,18,20,24,30$ and 36 and with bare couplings within the range $g_{0}^{2} \in[0.5,8]$. The measured couplings with the aforementioned parameters are shown in the left panel of Fig. 6 . It is clear from the figure that the finite volume effects become substantial on smaller lattices as the coupling grows larger. The measurements on the $L=36$ are incomplete and will not be included in the subsequent analysis.

Our data allows us to use either $s=2$ or $s=3 / 2$, and we choose the step size $s=3 / 2$. In the right panel of Fig. 6 we show the scaled step scaling function $\Sigma(u, L / a, 3 / 2) / u$ calculated for the measured pairs $8-12,12-18,16-24,20-30$ and $24-36$. The large coupling behavior of the $8-12$ pair deviates significantly from the others due to finite volume effects.

The continuum limit of the step scaling function is obtained from the lattice step scaling by extrapolating with a quadratic fit. The constant values of couplings at several lattice sizes are obtained by interpolating the measured couplings with a polynomial function

$$
\frac{g_{\mathrm{GF}}\left(g_{0}\right)}{g_{0}^{4}}-\frac{1}{g_{0}^{2}}=\sum_{i=0}^{m} a_{i} g_{0}^{2 i}, \quad m=10
$$



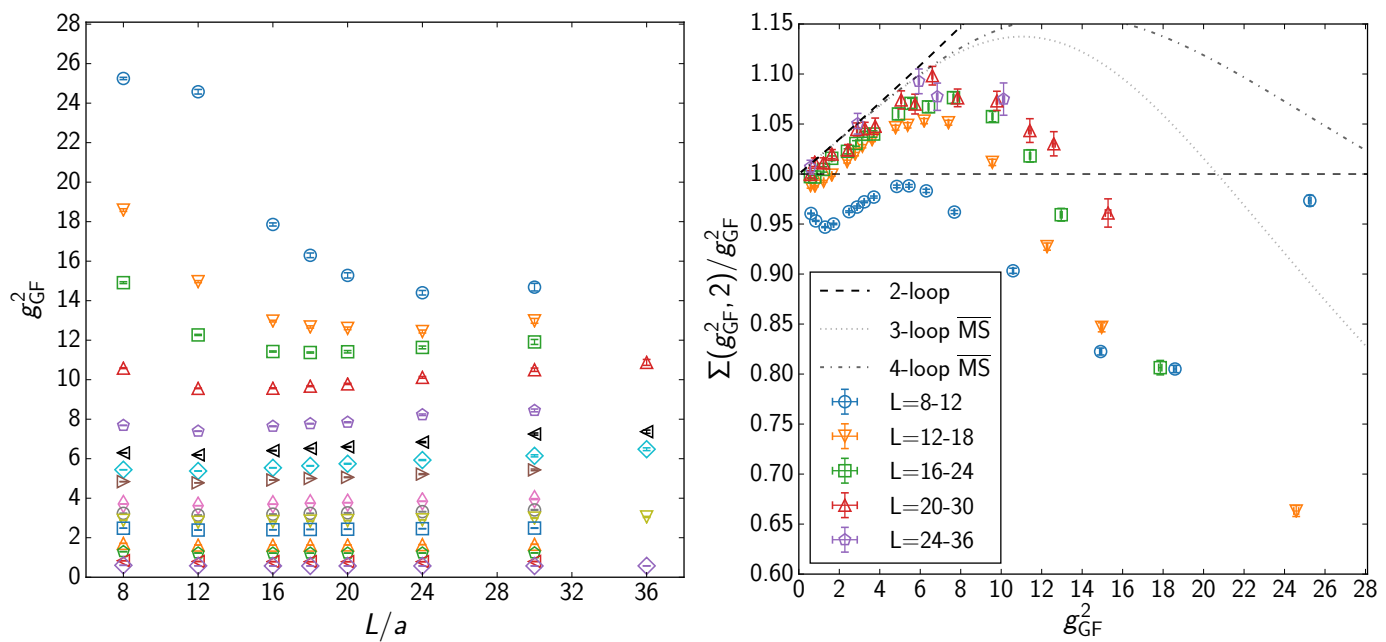

Figure 6. The plot on the left shows the gradient flow coupling (7) measured at each $\beta_{L}$ and $L / a$ at $c=0.3$ for the six flavor theory. The plot on the right shows the corresponding lattice step scaling function for these couplings.

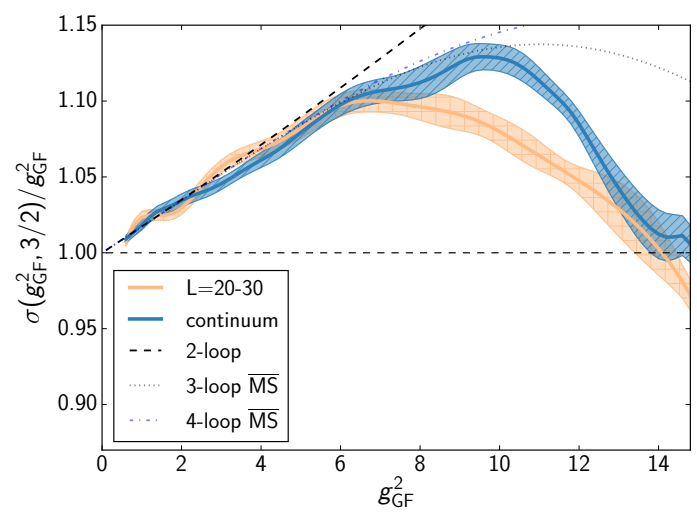

Figure 7. The scaled step scaling function with continuum extrapolation in the six-flavor theory.

with which we achieve a combined $\chi^{2} /$ d.o.f of $\sim 1.1$. Furthermore, the $\tau_{0}$-correction on the gradient flow coupling is implemented as explained in the case of the eight flavor theory.

We present a benchmark case of the continuum extrapolations of the step scaling function in the six flavor theory in Fig. 7. Similar to lattice step scaling behavior in Fig. 6, the continuum step scaling follows the universal two loop perturbative curve closely up to $g_{\mathrm{GF}} \approx 7$ and then deviates towards an IRFP around $g_{\mathrm{GF}} \sim 14.5$. The 3 and 4-loop $\overline{\mathrm{MS}}$ curves are also shown, but they are scheme dependent, and cannot be directly compared to the nonperturbative result. 


\section{Conclusions}

We have studied the infrared behaviors of SU(2) gauge theory with $N_{f}$ fermions in the fundamental representation of the gauge group. In particular, we have measured the running of the coupling in this theory with $N_{f}=8$ and $N_{f}=6$. The gradient flow algorithm with Schrödinger functional boundary conditions provides clean results on the strong coupling behavior of these theories. For both cases we have observed a clear indication of an infrared stable fixed point in the step scaling analysis. The continuum limit is robust regardless of the lattice discretizations used.

\section{References}

[1] A. J. Hietanen, J. Rantaharju, K. Rummukainen and K. Tuominen, JHEP 0905, 025 (2009).

[2] A. J. Hietanen, K. Rummukainen and K. Tuominen, Phys. Rev. D 80, 094504 (2009).

[3] J. Rantaharju, T. Rantalaiho, K. Rummukainen and K. Tuominen, Phys. Rev. D 93 no.9, 094509 (2016).

[4] H. Ohki et al., PoS LATTICE 2010, 066 (2010).

[5] F. Bursa, L. Del Debbio, L. Keegan, C. Pica and T. Pickup, Phys. Lett. B 696, 374 (2011).

[6] T. Karavirta, J. Rantaharju, K. Rummukainen and K. Tuominen, JHEP 1205, 003 (2012).

[7] M. Hayakawa, K.-I. Ishikawa, S. Takeda, M. Tomii and N. Yamada, Phys. Rev. D 88 no.9, 094506 (2013).

[8] T. Appelquist et al., Phys. Rev. Lett. 112 no.11, 111601 (2014).

[9] S. Capitani, S. Durr and C. Hoelbling, JHEP 0611, 028 (2006).

[10] T. DeGrand, Y. Shamir and B. Svetitsky, PoS LATTICE 2011, 060 (2011).

[11] M. Luscher and P. Weisz, Nucl. Phys. B 479, 429 (1996).

[12] M. Luscher, Commun. Math. Phys. 293, 899 (2010).

[13] P. Fritzsch and A. Ramos, JHEP 1310, 008 (2013).

[14] M. Luscher and P. Weisz, JHEP 1102, 051 (2011).

[15] M. Lüscher, JHEP 1008, 071 (2010).

[16] M. Luscher and P. Weisz, Commun. Math. Phys. 97, 59 (1985).

[17] M. Lüscher, JHEP 1406, 105 (2014).

[18] A. Ramos and S. Sint, Eur. Phys. J. C 76 no.1, 15 (2016).

[19] A. Cheng, A. Hasenfratz, Y. Liu, G. Petropoulos and D. Schaich, JHEP 1405, 137 (2014).

[20] A. Hasenfratz, D. Schaich and A. Veernala, JHEP 1506, 143 (2015).

[21] A. Ramos, PoS LATTICE 2014, 017 (2015). 